\title{
Exploration of bacterial community classes in major human habitats
}

\author{
Yanjiao Zhou ${ }^{1,4}$, Kathie A Mihindukulasuriya ${ }^{1}$, Hongyu Gao ${ }^{2}$, Patricio S La Rosa ${ }^{3}$, Kristine M Wylie ${ }^{1,4}$, John C Martin,
} Karthik Kota', William D Shannon ${ }^{3}$, Makedonka Mitreva', Erica Sodergren ${ }^{1,5}$ and George M Weinstock ${ }^{1,5^{*}}$

\begin{abstract}
Background: Determining bacterial abundance variation is the first step in understanding bacterial similarity between individuals. Categorization of bacterial communities into groups or community classes is the subsequent step in describing microbial distribution based on abundance patterns. Here, we present an analysis of the groupings of bacterial communities in stool, nasal, skin, vaginal and oral habitats in a healthy cohort of 236 subjects from the Human Microbiome Project.
\end{abstract}

Results: We identify distinct community group patterns in the anterior nares, four skin sites, and vagina at the genus level. We also confirm three enterotypes previously identified in stools. We identify two clusters with low silhouette values in most oral sites, in which bacterial communities are more homogeneous. Subjects sharing a community class in one habitat do not necessarily share a community class in another, except in the three vaginal sites and the symmetric habitats of the left and right retroauricular creases. Demographic factors, including gender, age, and ethnicity, significantly influence community composition in several habitats. Community classes in the vagina, retroauricular crease and stool are stable over approximately 200 days.

Conclusion: The community composition, association of demographic factors with community classes, and demonstration of community stability deepen our understanding of the variability and dynamics of human microbiomes. This also has significant implications for experimental designs that seek microbial correlations with clinical phenotypes.

\section{Background}

Knowledge of the composition, distribution and variation of bacteria in the human body has grown dramatically in the past decade. Different human habitats are composed of distinct microbial populations [1-8]. The range of abundance of components of the human microbiome extends over many orders of magnitude [9]. Inter-subject variation in bacterial community structure is also extensive in healthy humans $[4,7]$. Determination of the extent of the variability of the human microbiome is, therefore, crucial for understanding the microbiology, genetics, and ecology of the microbiome as well as for practical issues in experimental design and interpretation of clinical studies. In addition, the human microbiome is subjected to a continual flux

\footnotetext{
* Correspondence: george.weinstock@jax.org

${ }^{1}$ The Genome Institute, Washington University, St Louis, MO 63108, USA

${ }^{5}$ Current address: The Jackson Laboratory for Genomic Medicine, c/o University of Connecticut Health Center, 263 Farmington Avenue. Administrative Services Building, Call Box 901, Farmington, CT 06030, USA Full list of author information is available at the end of the article
}

of organisms from air, food, and other sources, transfer of organisms between body habitats through routine activity, cyclical changes in the physiology of body habitats on daily, monthly, and other timescales, which create changing selective pressures for each organism. Thus, temporal changes in bacterial communities (community stability) are also an important component of microbiome variation.

Evaluation of inter-subject variation is the first step to understanding the bacterial distribution in the human population. Furthermore, categorization of subjects based on the similarity or dissimilarity of their microbiota into groups by clustering techniques will not only help to reveal the bacterial distribution pattern in the population, but also facilitate our understanding of the underlying causes or the clinical association of specific types of microbial distributions. Indeed, recent data suggest the feasibility of such operational clustering. Specifically, the vaginal flora of asymptomatic women identified five groups by hierarchical clustering [6,10]. The five groups 
were defined based on the species or genera they contained. Race was found to be associated with the groups. Similar studies on microbial populations in stool samples identified three enterotypes [11]. Another approach identified two stool enterotypes by $\mathrm{k}$-means clustering and found that long-term diet was associated with enterotypes [12], which emphasized the biological significance of these enterotypes. Old Amish stool microbiota is disproportionally of the Prevotella enterotype [13]. Enterotypes have been discovered not only in humans, as recent studies have described two and three enterotypes in mice and chimpanzees, respectively, which resembled the human stool enterotypes $[14,15]$.

Several studies have not favored the enterotype concept [16-18]. Those studies focused on the stool microbiome distribution pattern in the human population, concluding that the stool microbiota was not a discrete distribution (three or two enterotypes) but rather a smooth gradient. Another issue is the appropriate number of clusters in the enteric bacterial community, for example, two or three stool enterotypes. These discussions largely emphasize the technical challenges in clustering data, and de-emphasize the value of categorization, namely, to codify and simplify relationships in a complex system and explore sensible biological groupings. A recent investigation on enterotypes across all the human body using the Human Microbiome Project (HMP) data showed that the enterotypes were affected not only by the data structure, but also by the methods applied in the clustering, such as clustering algorithms and distance measures [17]. These issues of clustering methodology are not surprising, since similar issues were previously seen in comparisons of clustering approaches for microarray data [19-21].

Because previous enterotype analysis of the HMP data is more technically orientated [17], biological inferences from the categorization are limited. Here, we used HMP 16S rRNA gene data from over 200 subjects, 18 body sites and two time points to interrogate the associated biology and explore the potential underlying mechanisms of the groups generated by two widely used clustering approaches. Because 'enterotype' originally referred to the microbiota type in stool, here we use an ecological term, 'community class', to refer to the clusters we identified in different habitats based on our cluster identification criterion, and use the generic term 'cluster' to refer to the groups of bacteria that do did not meet our criterion. We have identified three stool enterotypes and various community classes in the other 17 body habitats from the HMP 16S rRNA gene data and metagenomic shotgun data. We found association of demographic factors with different community classes. Also, for the first time we systematically assessed the stability of community classes and compared the subject composition in each community class from different habitats.

\section{Results}

Identification of community classes in human microbiota

Currently, there is no uniform statistical approach to determine the presence and optimal number of clusters or community classes from metagenomic samples $[6,11]$. Multiple approaches are recommended because the clustering approach is sensitive to the data sets [17]. We explored the community classes in each body site using both hierarchical clustering (complete linkage) and fuzzy k-means clustering with Bray-Curtis distances. The optimal number of clusters for a given habitat was determined by the silhouette method, a criterion used to choose the optimal number of clusters in previous enterotype studies [10-12]. The silhouette value is a measure of within and between cluster similarities. The number of clusters with the highest silhouette value is the optimal number of clusters in a data set. Twelve of eighteen sites had equal or higher silhouette values using hierarchical clustering with complete linkage, compared with k-means clustering (Table S1 in Additional file 1). The cluster similarities between different approaches and linkages used for hierarchical clustering are summarized in Table S2 in Additional file 1.

Three community classes from stool were determined based on the averaged silhouette statistics (silhouette = 0.25 for three clusters). This clustering solution resulted in clusters of size $\mathrm{N}=128, \mathrm{~N}=15$, and $\mathrm{N}=66$, which correspond to the Bacteriodes, Prevotella and Ruminococcus enterotypes identified from 39 European subjects [11], referred to as MetaHIT, as illustrated by principal coordinate analysis (PCoA) (Figure 1). It should be noted that the silhouette value only differs by 0.04 between two and three enterotypes.

To determine the effect of sample size on the number of clusters, we randomly subsampled 50, 100, 150 and 180 samples 100 times from the total 209 stool samples and computed the frequency of 2, 3, 4, and 5 clusters as the optimal cluster number in hierarchical clustering. At the subject scale, 52 of 100 subsamplings supported 2 clusters as optimal. With increasing numbers of subjects the frequency that 3 clusters was the optimal number increased to 72 of 100 subsamplings (Figure S1 in Additional file 2). This suggests that sample size as well as subject composition affects the number of clusters.

To further test the reliability of the clusters generated, we used two external confirmation methods. First, we clustered our data using an alternative approach. The 209 HMP stool samples were clustered using k-medoids with Jensen-Shannon divergence. This approach was identical to the approach used by the MetaHIT group [11], and generated the three clusters they identified with the highest silhouette values $=0.17$ (Figure S2 in Additional file 2). The silhouette values for three clusters in the k-means clustering were less than those generated 

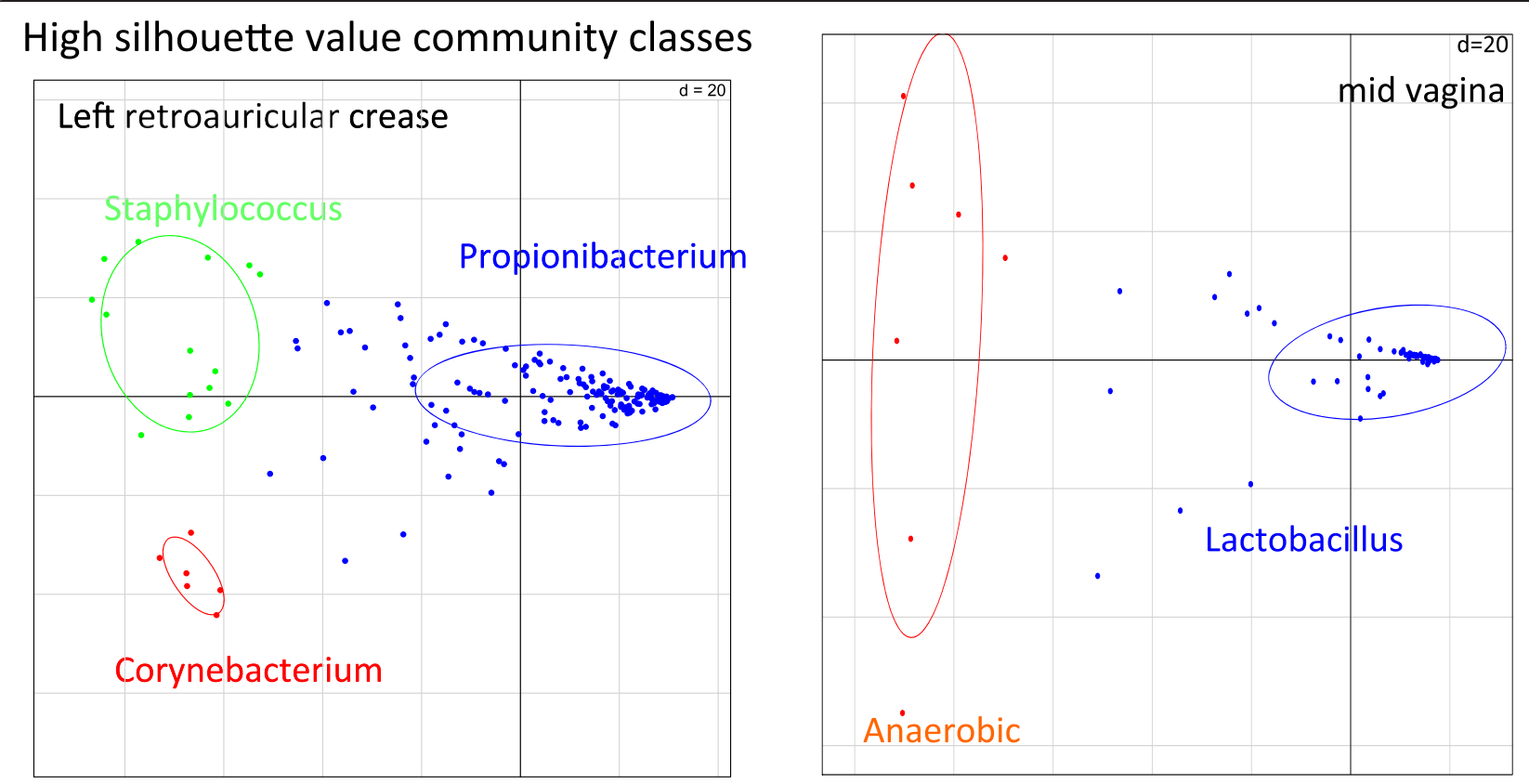

\section{Low silhouette value community classes}
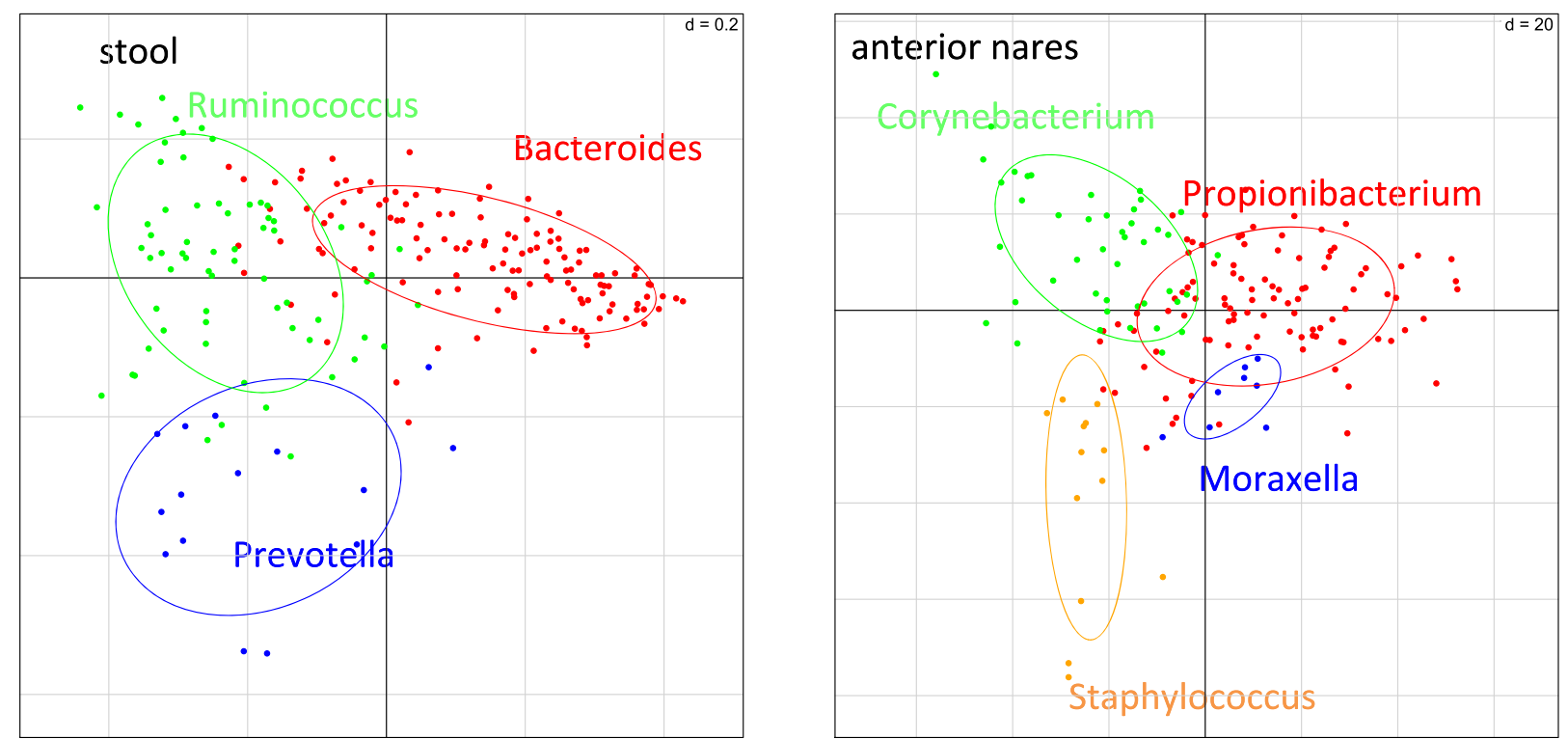

Figure 1 Examples of community classes of human body habitats as illustrated by PCoA. PCoA was used to visualize the community classes in different habitats. Samples are color-coded according to their community classes within the habitat. Only the major community classes from retroauricular crease are colored. The community classes show better separation in retroauricular crease and vaginal sites with higher silhouette values, and a less clear separation in stool and anterior nares with lower silhouette values.

by hierarchical clustering ( 0.17 versus 0.25$)$, suggesting that the latter technique performs better with the stool data type.

Although the same three enterotypes were generated by both clustering approaches, the prevalence of the specific enterotypes among the sampled subjects differed. Using hierarchical clustering, $61.2 \%, 31.6 \%$ and
$7.2 \%$ of the stool samples were assigned to Bacteroides, Ruminococcus and Prevotella community classes, respectively. Using the MetaHIT approach, 47.4\%, 42.1\% and $10.5 \%$ of the stool samples were grouped to the above community classes.

To further confirm the validity of the clusters, whole genome shotgun (WGS) sequencing was conducted on 81 
of the 209 samples. Applying our clustering/silhouette process to the metagenomic shotgun data recapitulated the same number and type of clusters as found with the larger sample size 16S rRNA gene and the MetaHIT data. Hence, the clusters were not due to bias from the amplification step of the 16S rRNA gene sequencing protocol.

Community classes were similarly determined for the rest of the 17 habitats. Silhouette values are similar for different numbers of clusters tested in the skin and vaginal sites (Table S1 in Additional file 1). For example, the average silhouette values for two to nine clusters in the right retroauricular crease ranged from 0.51 to 0.52 . Although the high silhouette values suggest that there were true clusters in this habitat, the optimal number of cluster was undetermined. The structure of this habitat always involved a single large cluster with high silhouette value and a variable number of smaller clusters (Figure S3 in Additional file 2), and the indeterminacy was due to estimating the number of these smaller clusters. Manual inspection of clusters determined the optimal number of clusters under this condition: placement of 158 samples from the right retroauricular crease in one cluster produced a very high silhouette value (0.59), while placement of 31 samples in the other cluster resulted in a silhouette value of 0.15 . Further dividing of the 31 samples into two clusters resulted in much higher silhouette values $(0.49)$ for the 19 samples in the first cluster, but an even lower value for the 12 remaining subjects (0.007). The latter value suggests that the bacterial community structures from the group of 12 subjects were very heterogeneous, prompting assignment of these samples as different clusters. Based on the above inspection of the silhouette values from individual groups and the taxonomic profiling from the dendrogram (Figure S6 in Additional file 2), we chose six clusters as the optimal number for the right retroauricular crease. Most subjects had high silhouette values for the six clusters, suggesting the cluster solutions are appropriate. A subset of samples had negative silhouette values, indicating improper grouping. For example, the subject with a negative silhouette value at the top of Figure S3 in Additional file 1 was grouped into Staphylococcus community classes with $16 \%$ of the Staphylococcus and $68 \%$ of Helicobacter. The relative abundances of Helicobacter in the rest of the subjects are low $(<1 \%)$; thus, this subject is inappropriate to be grouped in any clusters. In general, subjects with negative silhouette values are regarded as outliers. These subjects with unique bacterial community structure are not surprising considering the heterogeneity of the skin microbiota. Manual inspection, following the logic described above, was necessary for the three vaginal sites and four skin sites where silhouette values were similar between two or more clusters.
Table 1 summarizes the optimal number of community classes, corresponding silhouette values, and the number of subjects in each community class. Silhouette values for the optimal number of clusters vary by habitat. Three to six classes for skin sites and two to three classes for the vagina were identified with high silhouette values $(>0.5)$, indicating that community classes at these sites were well-defined (Figure 1) [22]. We also identified the five community classes (four driven by the genus Lactobacillus and one by anaerobic genera) in a subset of posterior fornix samples using WGS data, as previously reported $[6,10]$. Strain level analysis achieved finer resolution. The Lactobacillus gasseri group was divided into two subgroups occupied by the same species but different strains of L. gasseri (Figure S4 in Additional file 2). Interestingly, two anaerobic community classes were identified in the posterior fornix: Gardnerella dominated one community class, while Prevotella and Atopobium dominated the other. Although it was well known that all three genera were associated with vaginosis, our result calls attention to further categorization of these bacteria in healthy subjects as well.

The remaining habitats presented relatively low silhouette values. Four community classes were identified from 166 anterior nares samples (silhouette $=0.24$; Figure 1 ) . Except for keratinized gingiva, buccal mucosa and hard palate, the silhouette values in the rest of the oral sites were $<0.2$, suggesting that the bacterial communities in these habitats were more homogenous [23]. However, biologically interesting community classes were identifiable in some of the habitats with silhouette values $<0.2$, as addressed below.

The number of community classes was not consistent among similar sites. Symmetric sites, the left and right retroauricular creases, have three and six community classes, respectively, while the left and right antecubital fossas have five and six community classes, respectively. However, both retroauricular crease sites contained the same dominant community classes defined by Corynebacterium, Staphylococcus and Propionibacterium. The difference in the less dominant community classes is partly because the source subjects for the left and right site samples did not completely overlap. The high degree of inter-variation among the skin microbial community can produce some unique community classes in a small subset of subjects. The three vaginal sites are proximal, but contained different numbers of community classes. Most subjects were dominated by Lactobacillus. A small proportion of the subjects were dominated by one or two groups of anaerobic genera.

Habitat classification and indicator taxa of community classes In the high silhouette value habitats $(>0.5)$, such as the retroauricular crease, many samples from the main community class (Propionibacterium community class) were 
Table 1 Summary of community classes and their stability in human habitats

\begin{tabular}{|c|c|c|c|c|c|c|}
\hline Habitat type & Habitats & Silhouette value & Clusters & Membership & Dominant genera & ARI for two visits \\
\hline \multirow[t]{5}{*}{ Type I } & Posterior fornix & 0.86 & 3 & $3 / 5 / 80$ & Gardnerella / Prevotella / Lactobacillus & 0.57 \\
\hline & Mid-vagina & 0.78 & 2 & $7 / 81$ & Anaerobic genera / Lactobacillus & 0.58 \\
\hline & Vaginal introitus & 0.66 & 2 & $13 / 67$ & Anaerobic genera / Lactobacillus & 0.44 \\
\hline & Left retroauricular crease & 0.6 & 3 & $6 / 14 / 158$ & $\begin{array}{l}\text { Corynebacterium / Staphylococcus / } \\
\text { Propionibacterium }\end{array}$ & 0.56 \\
\hline & Right retroauricular crease & 0.52 & 6 & $4 / 4 / 4 / 4 / 19 / 154$ & 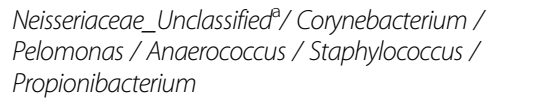 & 0.47 \\
\hline \multirow[t]{13}{*}{ Type II } & Left antecubital fossa & 0.30 & 5 & $2 / 6 / 7 / 28 / 30$ & $\begin{array}{l}\text { Sporacetigenium / Staphylococcus / Ralstonial } \\
\text { Propionibacterium / Conynebacterium }\end{array}$ & 0.07 \\
\hline & Right antecubital fossa & 0.37 & 6 & $3 / 3 / 4 / 6 / 8 / 61$ & $\begin{array}{l}\text { Streptophyta / Corynebacterium / Staphylococcus / } \\
\text { Streptococcus / Haemophilus / / Propionibacterium }\end{array}$ & 0.16 \\
\hline & Keratinized gingiva & 0.32 & 2 & $59 / 140$ & Prevotellaceae_Unclassified / Streptococcus & 0.37 \\
\hline & Buccal mucosa & 0.28 & 2 & $32 / 153$ & Haemophilus / Streptococcus & 0.12 \\
\hline & Hard palate & 0.25 & 2 & $10 / 183$ & Veillonella /Streptococcus & 0.04 \\
\hline & Anterior nares & 0.24 & 4 & $8 / 13 / 44 / 101$ & $\begin{array}{l}\text { Moraxella / Staphylococcus / Propionibacterium / } \\
\text { Conynebacterium }\end{array}$ & 0.26 \\
\hline & Stool & 0.25 & 3 & 15/66/128 & Prevotella / Ruminococcaceae / Bacteroides & 0.26 \\
\hline & Tongue dorsum & 0.18 & 3 & $35 / 77 / 94$ & NA & \\
\hline & Subgingival plaque & 0.21 & 2 & $97 / 104$ & NA & \\
\hline & Throat & 0.19 & 2 & $91 / 93$ & NA & \\
\hline & Supragingival plaque & 0.15 & 2 & $97 / 111$ & NA & \\
\hline & Palatine tonsils & 0.17 & 2 & $96 / 103$ & NA & \\
\hline & Saliva & 0.14 & 2 & $89 / 93$ & NA & \\
\hline
\end{tabular}

${ }^{a}$ Neisseriaceae in retroauricular crease and Ruminococcaceae in stool are the dominant orders (not genera).

${ }^{\mathrm{b}} \mathrm{A}$ heterogeneous group.

ARI, Adjusted Rand Index; NA, not applicable (indicator genera are not shown because of very low silhouette value).

tightly clustered, while the remainder of the samples formed smaller community classes, in which only a few subjects were included (Figure 1). On the other hand, in community classes in the relatively low silhouette value habitats $(0.25$ to 0.5$)$, such as stool and anterior nares, samples were less tightly clustered than for the high silhouette value habitats.

To examine the structure underlying different community classes, we correlated the alpha diversities (number of taxa within a sample) and the silhouette values in the 18 habitats. As indicated by Figure S5 in Additional file 2 , silhouette values are strongly negatively correlated with the Shannon diversities (Pearson correlation = 0.96). In particular, the two lowest alpha diversity habitats, vagina and retroauricular crease, had the highest silhouette values of the clusters, and the high alpha diversity habitats (saliva) exhibited low average silhouette values of its clusters. Based on the alpha diversity and silhouette values, the 18 habitats were divided into two types: type I (low diversity with median Shannon index $<1.5$ and high silhouette value) and type II (high diversity and low silhouette values) habitats. Type I habitats with low alpha diversity (Figure 1) were dominated by one genus. For example, Lactobacillus was present in each of the vaginal samples with an average abundance of $92 \%$ in the tightly clustered subgroup. Alternatively, bacterial communities in type II habitats were dominated by different genera to different degrees, leading to highly diverse communities. This diversity was reflected in the clusters, where samples were less tightly clustered than in type I habitats (Figure 1).

The definition of community class is based on the relative abundance of genera in bacterial communities. Certain key taxa are assigned as indicators, whose presence, absence, and relative abundance characterize that community class [24]. Indicator taxa were determined using the Dufrene-Legendre Indicator Species approach [25].

\section{Type I habitats}

The left and right retroauricular crease share three of the same three community classes (Propionibacterium, Staphylococcus, Corynebacterium) and most subjects were found in these three community classes. We used the left retroauricular crease as an example to show the indicator taxa that differentiate the community classes. 
While Propionibacterium is ubiquitously present on the skin of the healthy population, the median relative abundance is $86.5 \%$ in the Propionibacterium community class, but only $8.3 \%$ and $11.4 \%$ in the other community classes (Figure 2A). Likewise, the Staphylococcus community class features high abundance Staphylococcus (median relative abundance 55.0\%), compared with $6.4 \%$ and $19.8 \%$ in the other community classes. The median relative abundance of Corynebacterium is $41.7 \%$ in the Corynebacterium community classes and $0.6 \%$ and $10.8 \%$ in other classes. Differentiation of community classes also involves less abundant taxa. In total, 14 taxa were significantly different between the community classes of the left retroauricular crease samples $(P<0.01$; Table S3 in Additional file 1).

Using mid-vagina as a vaginal habitat representative, we determined indicator genera for the vaginal community classes. The relative abundance of Lactobacillus ranges from $31.3 \%$ to $99.9 \%$ in the Lactobacillus community class. In the anaerobic community class, Lactobacillus is less abundant while the anaerobic genera, such as Prevotella, Sneathia, Bifidobacterium, Megasphaera, Dialister, and Atopobium, are more abundant (Table S3 in Additional file 1). These genera are reported to be vaginosis-associated genera [26].

\section{Type II habitats}

The top four most abundant genera in the anterior nares are Corynebacterium, Propionibacterium, Staphylococcus, and Moraxella, each of which is the dominant genus for a distinct community class (Figure 2B; Table S3 in Additional file 1). Each of these genera contains pathogenic species, suggesting that the anterior nares is a potential reservoir for pathogens.

Characterization of anterior nares bacterial communities in 40 individuals by $16 \mathrm{~S}$ rRNA fingerprinting based on single-strand conformation polymorphisms demonstrated 10 clusters with the majority of subjects (36 of 40) grouped into five clusters [27]. Four of these clusters were identified in our analysis, the exception being a Finegoldia group. In our $16 \mathrm{~S}$ rRNA gene data set Finegoldia was present in only one of the samples, and with very low abundance. Finegoldia is isolated most frequently from various infected sites [28], and is less common in healthy subjects.

Five and six community classes were identified in the left and right antecubital fossa samples, respectively. Three of these, dominated by Propionibacterium, Corynebacterium, and Staphylococcus, were also found in the retroauricular crease samples. However, Propionibacterium abundance in the Propionibacterium community class of antecubital fossa is lower than that in retroauricular crease (average of $61.6 \%$ of the community compared with 92\%), it is still 5-fold more abundant than in the other community classes. In total, 24 indicator genera were identified among the 7 community classes (Table S3 in Additional file 1).

In the MetaHIT study, the Ruminococcus type is the most frequent enterotype in stool, followed by Bacteroides and Prevotella [11]. In our analysis, most of the subjects were grouped to Bacteroides followed by Ruminococcus and Prevotella. The average abundance of Bacteroides in our data set is $55 \%$, in contrast to the average abundance of $35 \%$ in the other study (Figure 2C) [11]. There are methodological as well as demographic differences in the subjects in these studies, so the quantitative differences are not surprising.

It is difficult to delimit clusters in most oral sites due to the homogeneity of bacterial communities. We nevertheless observed biologically important groups in the subgingival plaque in this healthy cohort. We found that the subgingival plaque from 122 subjects was inhabited by periodontitis-associated genera such as Treponema and/or Porphyromonas with a median relative abundance of $4.3 \%$ and $3.9 \%$, compared with $0.05 \%$ and $0.73 \%$ in the rest of the subjects, respectively. Fourteen subjects exhibited significant amounts of Treponema and/or Porphyromonas, accounting for $26 \%$ to $44 \%$ of total bacteria in their subgingival plaque. Those subjects are major components of cluster 2 in Figure 2D. We use the generic term cluster in this context to distinguish the community classes described in other habitats where clusters have a higher silhouette value. On the other hand, the relative abundance of Veillonella, a genera that slows the development of dental caries [29], was very low in cluster 2 (Figure 2D). In the supragingival plaque, pathogenic anaerobes are less abundant compared with subgingival plaque because this is a less anaerobic environment compared with the subgingival region [30].

In keratinized gingivae, buccal mucosa, hard palate, palatine tonsils and throat, one community class was dominated by Streptococcus, and the other community class was dominated by genera varying with habitats (Table S3 in Additional file 1). Interestingly, 59 subjects had keratinized gingiva microbiome distinguished by a high abundance of unclassified Prevotellaceae and unclassified Bacteroidales; this community class represented a less characterized taxonomic group (Figure S6 in Additional file 2). Tongue dorsum and saliva are two habitats in which the Streptococcus abundance is less dominant than in other oral sites. Instead, Neisseria is an essential genus in one-third of tongue dorsum samples, resulting in detection of the Neisseria community class (Table S3 in Additional file 1; Figure S6 in Additional file 2).

\section{Community class comparisons across all habitats}

The HMP data sets not only provide the opportunity to characterize the community classes of multiple habitats, 


\section{A left retroauricular crease \\ Propionibacterium}

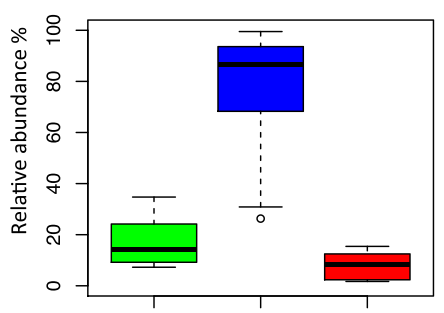

B anterior nares

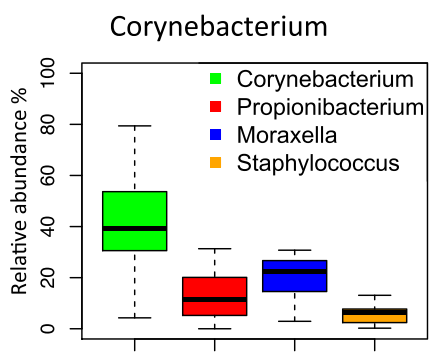

Staphylococcus

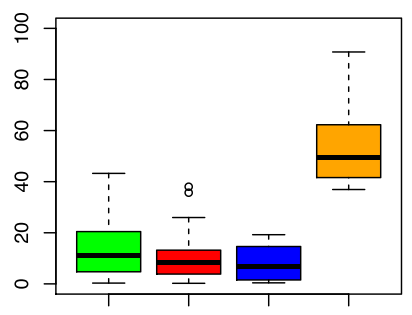

C stool

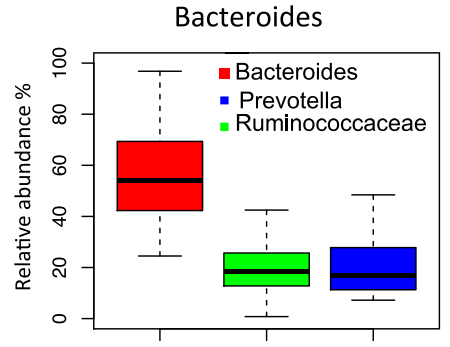

D subgingival plaque

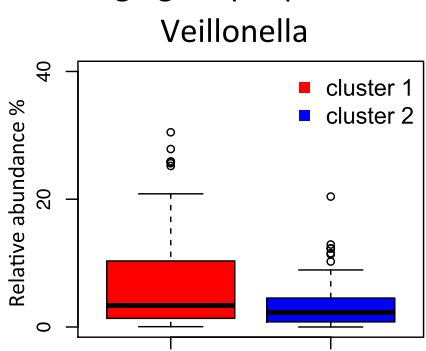

Staphylococcus

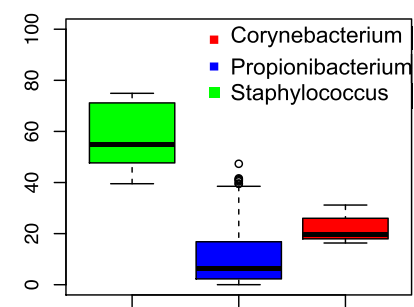

Propionibacterium

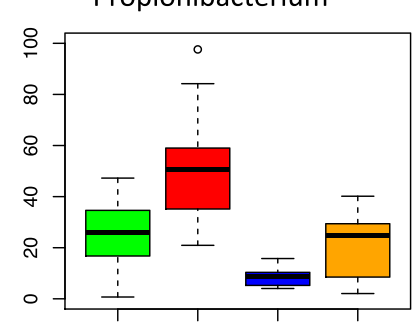

Corynebacterium

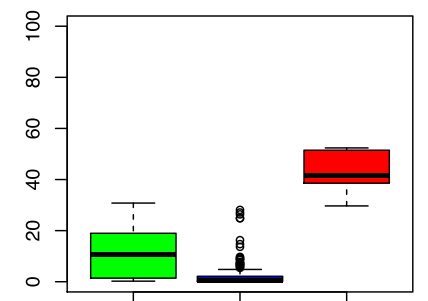

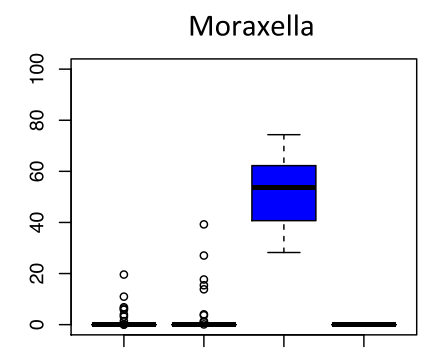

Ruminococcaceae(Oscillibacter)

Prevotella
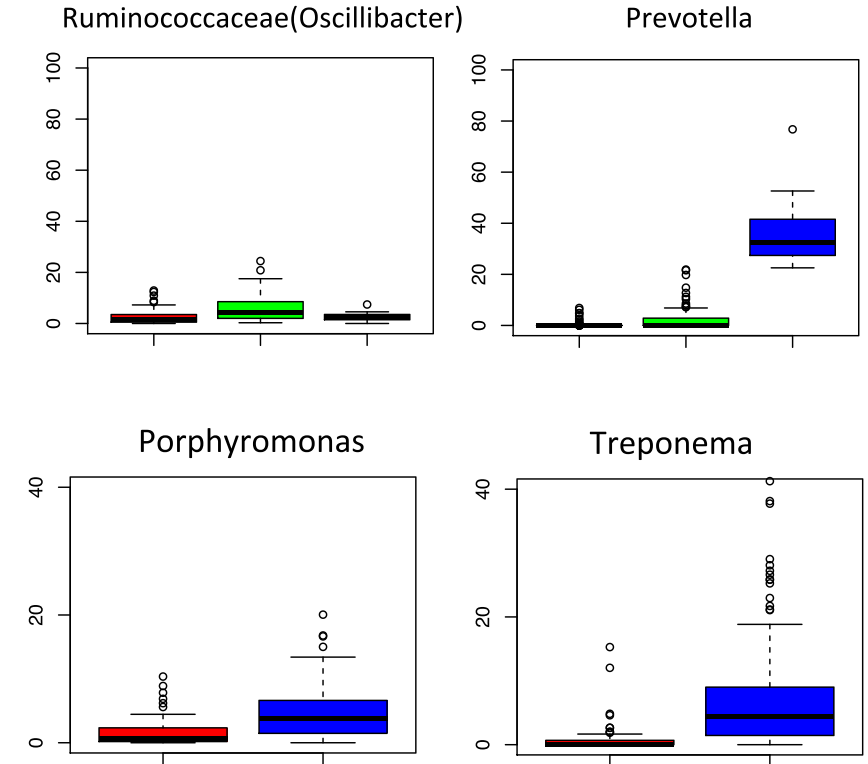

Figure $\mathbf{2}$ (See legend on next page.) 
(See figure on previous page.)

Figure 2 Examples of indicator taxa between community classes. Indicator taxa driving the differentiation of community classes were identified using the indval function in the labdsv package in R. Boxplots are labeled according to the dominant taxa in the community class. (A-D) The colors represent different community classes. The relative abundances of dominant taxa (y-axis) in each community class are plotted for the type I habitat left retroauricular crease (A), and the type II habitat anterior nares (B), stool (C), and subgingival plaque (D). In (D), the name of the community classes are designated as a generic term, cluster 1 or cluster 2 , because of the very low silhouette value $(<0.2)$.

but also allow us to determine if there are any correlations between community classes at different habitats in the same individual. This can be addressed by determining if a group of subjects who carry a particular community class in one habitat also belong to the same community class at other habitats.

To answer this question, we compared the subjects from different community classes in each pair of habitats. Subjects who had samples from both habitats were included in the clustering analysis. The Adjusted Rand Index (ARI) was used to assess the similarity between clusters in different habitats [31], where a comparison is made between the assignments of each pair of subjects in the clusters of the two habitats under comparison. Complete correlation between subjects at two habitats produces an ARI of 1. As shown in Figure 3, the subject composition in the community classes was highly consistent, with ARI $=0.64$ in the three vaginal sites. The left and right retroauricular creases were also similar when comparing subject compositions in the community classes (ARI $=0.32$ ). Other paired habitats showed low similarity for community classes (Figure 3 ).

Because the anterior nares, retroauricular crease, and antecubital fossa each have three community classes dominated by Propionibacterium, Corynebacterium, and Staphylococcus, we performed a detailed comparison of subjects carrying these three community classes. In particular, the Corynebacterium community class is the most common community class in the anterior nares, yet only $18 \%$ (16 of 87) of samples with the Corynebacterium community class in the anterior nares belonged to the Corynebacterium community class of antecubital fossa. In contrast, 50\% of the samples with the Corynebacterium community class in the anterior nares were assigned to the Propionibacterium community class of antecubital fossa. The rest were assigned to the Staphylocccus community class or other small classes in antecubital fossa. Moraxella was the fourth community class in anterior nares, and subjects with a high abundance of Moraxella in their anterior nares did not have high abundance Moraxella in their skin. Therefore, bacterial community structures across subjects are confined to a given habitat and the drivers of community structure act independently of other unrelated habitats.

\section{Associations of demographic factors with bacterial community structure}

The association of demographic factors (gender, geographical location, ethnicity, body mass index (BMI), age) with each community class was tested by Fisher's exact test or ANOVA, and $P$-values were corrected using the Bonferroni approach (Materials and methods; Table 2; Figure S6 in Additional file 2).

Gender was significantly different between the community classes in the retroauricular crease, antecubital fossa and anterior nares (Figure 4). In the retroauricular crease, the Staphylococcus community class was mainly carried by females in contrast to the relatively even gender distribution for Propionibacterium and Corynebacterium community classes $(P=0.0004$; Figure $4 \mathrm{~A})$. In the antecubital fossa, male samples were dominated by Propionibacterium and females by the Staphylococcus community class $(P=0.005$; Figure $4 \mathrm{~B})$. In anterior nares, female subjects had over-representation of Staphylococcus community classes in anterior nares, whereas the Moraxella community class was mainly composed of male subjects $(P=0.05$; Figure $4 C)$. As expected, the relative abundances of each genus described above in male and female subjects from skin and anterior nares sites were also significantly different (Figure S7 in Additional file 2).

Among the 15 subjects with the Prevotella enterotype in stool, 13 were male, which did not differ from the gender distributions of Bacteroides and Ruminococcus enterotypes after Bonferroni correction $(P=0.14)$. However, among samples in which Prevotella was present, we found that the median abundance of Prevotella in all the male samples was 10 -fold greater than in female samples $(P=0.005$; Figure S7 in Additional file 2$)$. We did not detect a correlation of BMI with any enterotypes or with the ratio of Bacteroidetes and Firmicutes in stool.

Consistent with prior studies [6], $\mathrm{pH}$ was strongly associated with the bacterial community structure in the vaginal sites (Table 2). $\mathrm{pH}$ was significantly higher in the anaerobic bacteria-dominant community class $(P=0.01)$. Ethnicity differs significantly $(P=0.04)$ in the Lactobacillus community class compared to the anaerobic bacteria subgroup.

The subjects in the Propionibacterium community class are about 4 years older than other community classes in retroauricular crease $(P=0.003$; Figure 4D). Age was also significantly different between community classes in hard palate $(P=0.02)$, as were site of residence (St Louis versus Houston) and race in oral sites (Table 2).

\section{Community class stability}

Figure 5A illustrates the community class changes between two time points using left retroauricular crease as 


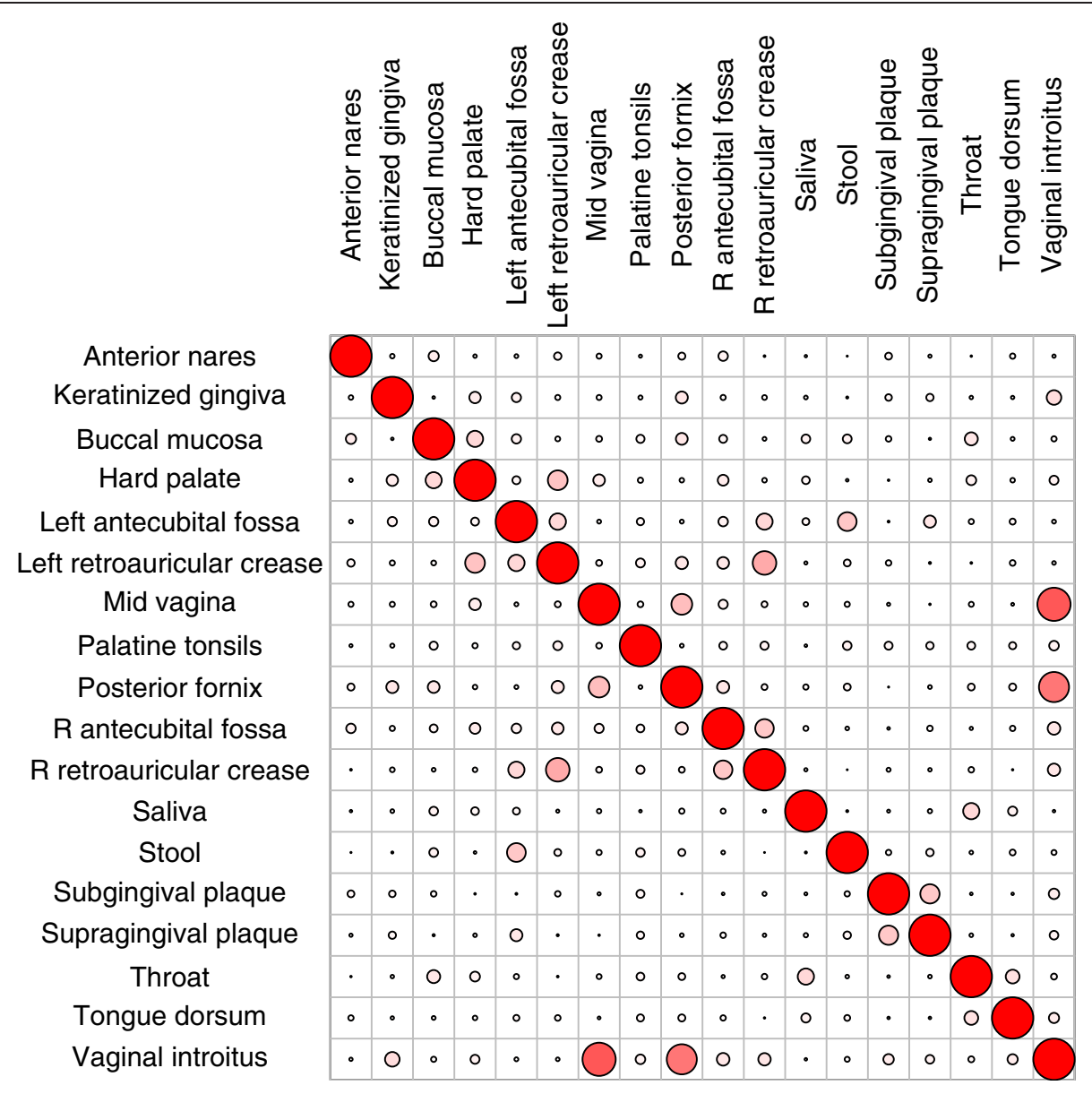

Figure 3 Community class comparisons across all habitats. Cluster similarity was analyzed using the ARI. A large overlap of carriers was detected between community classes in left and right retroauricular creases as well as within three vaginal sites. The color saturation and size of the circles represent the degree of cluster similarity. The circles along the diagonal from top left to bottom right, with an ARI equal to 1, indicate self-comparison.

an example. Three community classes were identified in left retroauricular crease in the first sampling and maintained in the second sampling. The ARI measuring the agreement of paired subjects in the clusters of two time points was 0.47 . In particular, $86 \%$ of the subjects were in the same community class on both visits. This relative stability is largely because of the stability of the major Propionibacterium community class. Of 44 subjects, 39 maintained the Propionibacterium community class (Figure 5A). One of three subjects from the Corynebacterium community class switched to the Staphylococcus class while the three subjects in the Staphylococcus class still clustered in the same class. One subject from the Propionibacterium and two subjects from the Corynebacterium class switched to an unclassified Neisseriaceae class at the second time point. Because each community class was defined by the relative abundance of the genera in the community, switching between community classes indicates a significant change in the abundance of genera.
We also evaluated community class stability in the vaginal introitus. The ARI was high (0.57) for community classes from the two samplings. Eighty-six percent of subjects retained the same community classes between visits. Most subjects (23 of 25 subjects) from the Lactobacillus community class stayed in the same community classes at the second visit. Two subjects switched to the anaerobic community class. Two of four subjects from the anaerobic community class in the first visit switched to the Lactobacillus class on the second visit (Figure S8 in Additional file 2). Subjects that switched from the Lactobacillus to the anaerobic community class showed increased vaginal $\mathrm{pH}$, which may be caused by the decrease in lactic acid bacteria. Overall, the community classes in type I habitats (retroauricular crease and vagina) tend to be stable over time because of the high abundance of single genera in their communities.

In contrast to the type I habitats, community classes in type II habitats appeared to be less stable over time. For this analysis, the left and right antecubital fossa data 
Table 2 Association of demographic factors with community classes

\begin{tabular}{|c|c|c|c|c|c|c|c|c|c|c|}
\hline \multirow[b]{2}{*}{ Habitats } & \multicolumn{2}{|l|}{ Gender } & \multicolumn{2}{|l|}{ Age } & \multicolumn{2}{|l|}{ Race } & \multicolumn{2}{|l|}{ Sites } & \multicolumn{2}{|l|}{ Vaginal PH } \\
\hline & $\begin{array}{l}\text { Community classes } \\
\text { for comparison }{ }^{a}\end{array}$ & $P^{\mathrm{b}}$ & $\begin{array}{l}\text { Community classes } \\
\text { for comparison }{ }^{\mathrm{a}}\end{array}$ & $P^{\mathrm{b}}$ & $\begin{array}{l}\text { Community classes } \\
\text { for comparison }{ }^{\mathrm{a}}\end{array}$ & $P^{\mathrm{b}}$ & $\begin{array}{l}\text { Community classes } \\
\text { for comparison }^{\mathrm{a}}\end{array}$ & $P^{\mathrm{b}}$ & $\begin{array}{l}\text { Community classes } \\
\text { for comparison }^{\mathrm{a}}\end{array}$ & $P^{\mathbf{b}}$ \\
\hline Anterior nares & $\begin{array}{l}\text { Propionibacterium } \\
\text { Corynebacterium, } \\
\text { Staphylococcus, } \\
\text { Moraxella }\end{array}$ & 0.05 & & & & & & & & \\
\hline Antecubital fossa & $\begin{array}{l}\text { Staphylococcus, } \\
\text { Propionibacerium }\end{array}$ & 0.005 & & & & & & & & \\
\hline Retroauricular crease & $\begin{array}{l}\text { Staphylococcus, } \\
\text { Propionibacterium, } \\
\text { Conynebacterium }\end{array}$ & 0.0004 & $\begin{array}{l}\text { Propionibacterium } \\
\text { non-Propionibacterium }\end{array}$ & 0.003 & & & & & & \\
\hline Hard palate & & & Veillonella, Streptococcus & 0.02 & & & & & & \\
\hline Buccal mucosa & & & & & & & Haemophilus Streptococcus & 0.00005 & & \\
\hline Subgingval plaque & & & & & & & Two clusters & $8.0 \times 10^{-10}$ & & \\
\hline Supragingival plaque & & & & & Two clusters & 0.03 & Two clusters & $3.8 \times 10^{-7}$ & & \\
\hline Throat & & & & & Two clusters & 0.04 & Two clusters & $6.2 \times 10^{-6}$ & & \\
\hline Palatine tonsils & & & & & Two clusters & 0.03 & Two clusters & $9.5 \times 10^{-7}$ & & \\
\hline Saliva & & & & & Two clusters & 0.03 & Two clusters & 0.00005 & & \\
\hline Vagina & & & & & Lactobacillus, anaerobic class & 0.04 & & & Lactobacillus, anaerobic class & 0.01 \\
\hline
\end{tabular}

${ }^{\mathrm{a}}$ Community classes used in the demographic association analysis.

b-values after Bonferroni correction.

Empty fields indicate there is no significant difference between the community classes for a give demographic factor. 


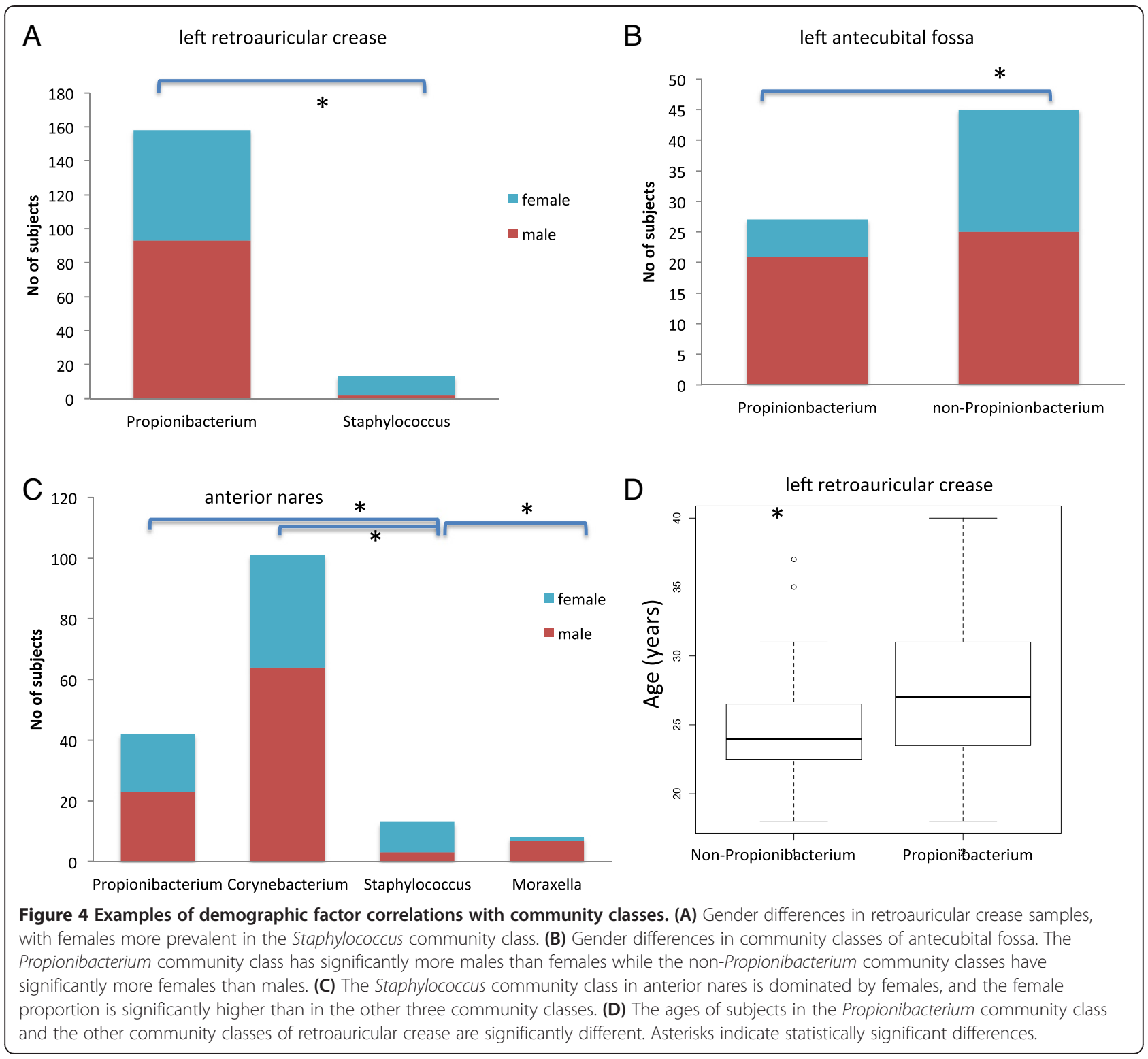

were combined at each time point because of the small amount of repeat data available. The ARI was 0.2 for the antecubital fossa. Of 33 subjects, 20 (60\%) maintained their community classes at the second visit (Figure S8 in Additional file 2). The less dominant community under active switching represented the less stable community class. Likewise, 22 of 48 sample pairs (45\%) switched their anterior nares class at the second visit $(A R I=0.26)$, indicating the dynamic nature of the bacterial community in the anterior nares (Figure 5B). Thirty-two percent of subjects switched from Corynebacterium to Propionibacterium and $24 \%$ of Propionibacterium subjects switched to Corynebacterium. Subjects also switched in both directions between the Corynebacterium and Moraxella classes. Switching also was observed from Propionibacterium to Staphylococcus, Staphylococcus to Corynebacterium, and Moraxella to Staphylococcus. We did not observe switching from the Staphylococcus to the Propionibacterium or Moraxella classes. This may be because of the small sample size (two subjects) for the Staphylococcus community class. Also, it will be interesting to probe the role of gender barriers in the transition between community classes because male and female subjects were dominated by different community classes.

Switches between stool community classes (31\%) were more frequent than in type I habitats, but less frequent than in other type II habitats (ARI 0.26). The switching mainly occurred between the Bacteroides and Ruminococcus community classes (Figure 5C). Although Bacteroides dominates in the Bacteroides community class, a subset of subjects had high relative abundance of Ruminococcus. These subjects tended to convert to the Ruminococcus 


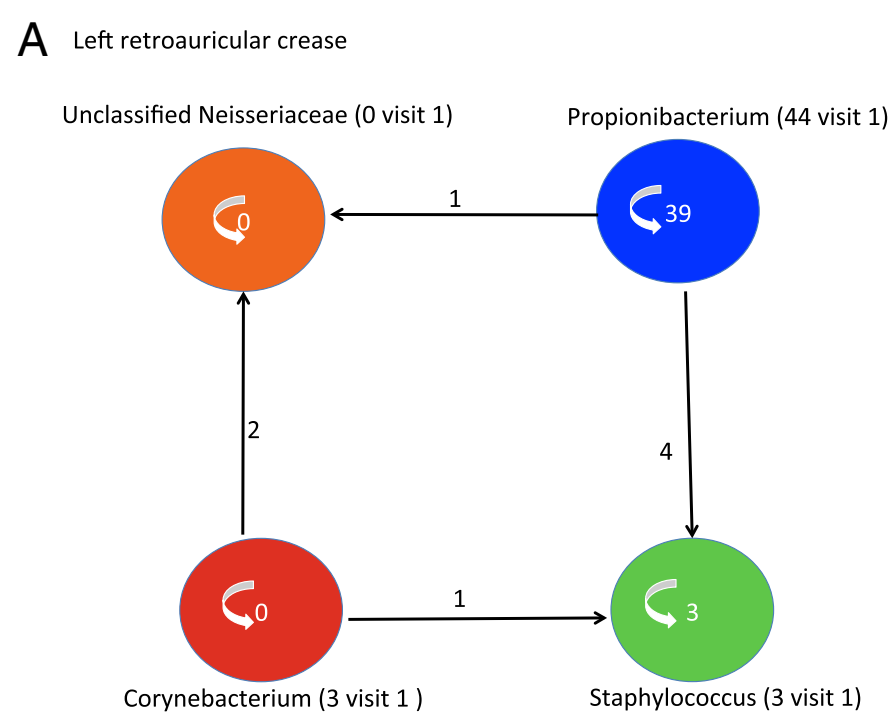

B Anterior nares

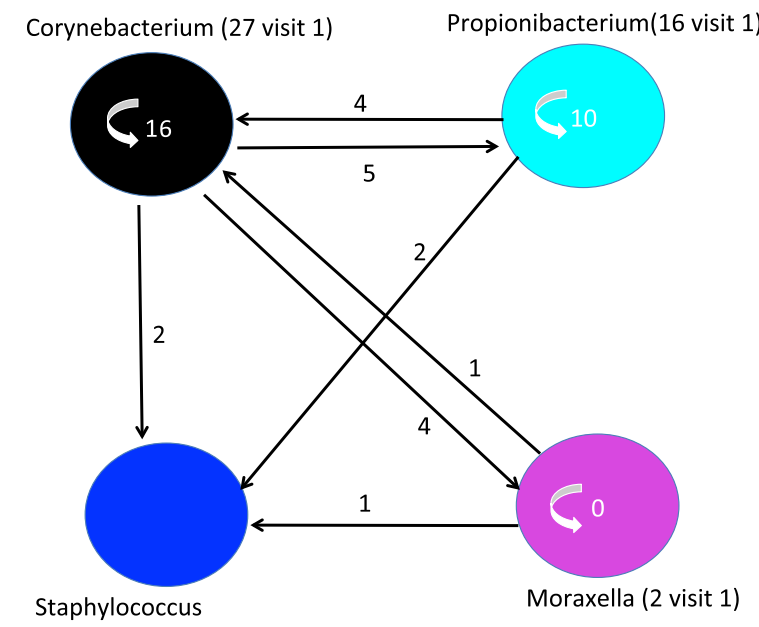

C stool

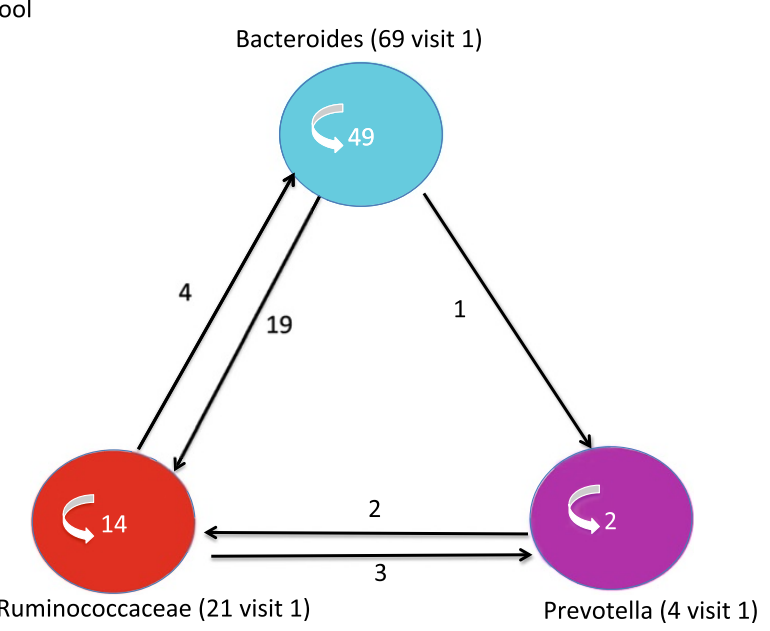

Figure 5 (See legend on next page.) 
(See figure on previous page.)

Figure 5 Dynamics of community classes over time. (A-C) Subjects from different community classes switch to other community classes or maintain their original community class in different habitats: (A) retroauricular crease, (B) anterior nares, and (C) stool. The colored circles represent different community classes. The names of community classes are followed by the number of subjects belonging to the community classes at the first visit. Subjects transferring to other community classes at the second visit are indicated by arrows along with the number of subjects that switched. The number inside each community class indicates the number of subjects maintaining the same community class at the second visit.

community class. This is also true for subjects belonging to the Ruminococcus community class but with a relatively high abundance of Bacteroides. These subjects tended to convert to the Bacteroides community class at the second visit.

As addressed above, community classes in most oral habitats are not as distinct as type I habitats, the active conversion between classes reflecting the uncertainty of the clusters in those habitats except saliva, tongue dorsum and keratinized gingiva (Table 1).

We additionally examined the variation of Treponema between visits in subgingival plaque. The 14 subjects with high relative abundances of Treponema (26\% to 44\%) at the first visit retained 7- to 8-fold higher than average abundance of Treponema at the second visit. The stability of the high abundance Treponema phenotype between visits in a subset of the subjects shows that the predominance of Treponema in these individuals is not a transient event, reinforcing the concept that these subjects are at higher risk to develop periodontal disease.

\section{Discussion}

Reports on the three stool enterotypes sparked an ongoing debate on whether stool microbiota is actually discrete or a continuum [16]. Enterotypes have been reported in humans and other animals [12,14,15]. There has been controversy regarding the conceptual appropriateness of discrete or categorical enterotypes versus a population description based on a gradient pattern of bacterial community structure [18]. The debate has been focused largely on the clustering techniques [17] and the assignment of the number of clusters in the community. However, these concerns overlook the purpose of the categorization of the microbiota in the human body, that is, determination of like versus not-like groupings of organisms, and easily conveyed descriptions of populations with which to find biologically meaningful groups. Clustering simplifies the complex relationships between objects and the cluster solutions vary with different distance/dissimilarity measurements and clustering algorithms $[17,20,21]$. Clustering is only an exploratory technique and should not play a decisive role in data analysis.

Our analysis of stool identified three stool enterotypes, consistent with previous studies. A study using the HMP metagenomic data indicated only two clusters for each of the 18 body sites, including stool [17]. Our analysis identified two clusters for oral and two vaginal sites, but more in stool and skin habitats. We further manually inspected the hierarchical cluster solutions in skin and vaginal sites where the silhouette values were similar for different numbers of clusters. This manual inspection resolved more biologically interesting clusters. This manual inspection is based on subjective interpretation and related biological knowledge is thus required. For example, our inference of two community classes in the posterior fornix was based on our findings from an independent project where race differed in different anaerobic groups (data not shown). Overall, the clustering analysis serves as a starting point for assessing the existence and number of the groupings, and obligate further investigation to determine biological validity.

Recent work has shown the effect of diet on enterotypes [12]. Specific long-term diets, especially those high in protein and fat, were linked to Bacteroides enterotypes, while carbohydrates were linked to Prevotella enterotypes $[12,15]$. The predominance of the Bacteroides enterotype identified in the HMP healthy cohort may reflect the natural presence of enterotypes in St Louis and Houston populations on American diets. Our findings might have clinical relevance. For example, metabolic diseases such as obesity are associated with the elevation of a wide range of cytokine and inflammation markers. Calprotectin, a gut inflammation marker, is higher in mice that harbor a Bacteroides enterotype $[12,15]$, suggesting that this genus triggers low-grade inflammation reactions in the gut. The relationships of enterotypes, diet and inflammation raise the possibility of manipulating gut enterotypes to mitigate risk of metabolic diseases.

Subjects from the HMP cohort are healthy as defined by clinical criteria; we nevertheless detected signals for disease. For example, a cluster dominated by periodontitisassociated genera such as Treponema and/or Porphyromonas was identified in subgingival plaque samples. Treponema is considered a major etiological bacteria in periodontitis and Porphyromonas is strongly associated with chronic adult periodontitis [30,31]. Treponema is a genus consisting of many species, but the Treponema species are rare in healthy subjects compared with subjects with periodontal disease [32]. The high abundance of periodontal disease-associated genera and low abundance 
of protective genera in the healthy population is an example of the pathogenic bacterial load carried by healthy individuals. Similarly, the anaerobic group was identified in a small subset of the vaginal samples from this cohort. A high abundance of anaerobic genera in vagina is often linked to vaginosis. The identification of an anaerobic group in the HMP cohort may be a result of the inclusion of unhealthy individuals in the cohort, because of the incomplete criterion used for the diagnosis of bacterial vaginosis [32]. Alternatively, it may represent a normal vaginal flora for non-Caucasians [6].

Phenotypic characterization of community classes also sheds light on mechanisms underlying these differences. Gender correlated with community classes in the skin, anterior nares and stool. What drives the difference between male and female skin and nasal community composition? Intrinsic properties of bacteria and physiological differences between genders may contribute to the microbiota composition difference. Male skin has more collagen and sebum with larger pores, a richer blood supply, and an increased tendency to sweat $[23,33]$. Thus, male skin may provide more nutrition for the two slowgrowing genera, Propinionbacterium and Corynebacterium, that are favored among men in this cohort. The different bacterial compositions of male and female skin might also lead to phenotypic differences. For example, odor precursors in men and women's sweat are modulated by the gender differences in bacterial compositions [34]. We found significantly more male subjects in the Prevotella community class before statistical correction, although this becomes insignificant after correcting for multiple comparisons. Human microbial communities interplay with both environmental and host factors, so the community class pattern may result from the combined influence of both endogenous and exogenous factors.

The habitat-specific community classes for subjects are dynamic. This includes differences based on age [35], point in menstrual cycle [36,37], changes in health states and other lifetime events. It has been reported that bacterial abundance can vary over short periods [38]. We observed that community class stability is habitatdependent, with the conversion between community classes being more common in type II habitats. It is noteworthy that for those habitats with relatively welldefined classes (skin, vagina, anterior nares and stool), switching mainly occurs in minor community classes whereas dominant community classes maintain dominance over time. The fundamental mechanism of conversion between community classes is not known. Short-term diet can change the microbial composition and abundance, but has not been shown to lead to the replacement of enterotypes [12]. Delimitation of the genetic demographic, environmental, behavioral and nutritional factors that influence community classes in humans is challenging.
Animal experiments in a well-controlled setting will be an attractive approach to address to what extent the above factors or a combination of multiple factors contribute to the formation of and changes between community classes. This information will provide significant value in how to alter the human microbiome to prevent or treat disease in the future.

\section{Conclusion}

We identified 2 to 6 community classes for each of the 18 habitats from the HMP healthy cohort by clustering augmented by manual inspection. These community classes are associated with a number of host factors, including gender, race, age and geography, suggesting that the identification of the community classes is non-random. The dynamics of the community classes over a year-long interval underscores the complex interplay of our microbiota with the internal and external environment.

\section{Materials and methods Ethics statement}

Subjects provided written informed consent for screening, enrollment and specimen collection. The protocol entitled 'HMP-07-001 Human Microbiome Project Core Microbiome Sample Protocol A' was reviewed and approved by institutional review board at Washington University in St Louis, IRB ID\#: 201105198 (previously 08-0754) and Baylor College of Medicine, IRB ID\#: $\mathrm{H}-22895$. The data were analyzed without personal identifiers. Research was conducted according to the principles expressed in the Declaration of Helsinki.

\section{Sample collection}

Specimens were collected by teams at the Baylor College of Medicine and Washington University in St Louis [32]. In total, 236 healthy adults were included in this analysis. Fifteen habitats comprising anterior nares, skin (left and right retroauricular crease, left and right antecubital fossa), oral (hard palate, keratinized gingiva, buccal mucosa, subgingival plaque, supragingival plaque, saliva, tongue dorsum, palatine tonsil and throat) and stool were sampled from all subjects. Female subjects were sampled at three extra sites: vaginal introitus, posterior fornix and mid-vagina. For longitudinal studies, a set of samples from each habitat was collected at two time points (visit one and visit two) separated by 30 to 359 days.

\section{DNA sequencing, quality control and taxonomic classification} To analyze the $16 \mathrm{~S}$ rRNA gene, the V3-5 region of the $16 \mathrm{~S}$ RNA gene was sequenced on the Roche-454 platform to define the composition of the bacterial community. Sample preparation, DNA isolation, sequencing, and data processing were performed following the standardized protocols 
developed by the HMP consortium [39]. In brief, data processing allowed one mismatch in the barcode and up to two mismatches in primer. The minimal acceptable sequence length was $200 \mathrm{bp}$.

This dataset is the July $201016 \mathrm{~S}$ rRNA gene sequencing data freeze, 7,518 SRA runs, the Human Microbiome Project $16 \mathrm{~S}$ rRNA Clinical Production Phase I, available from NCBI at [40], and from the HMP Data Analysis and Coordinating Center at [41].

These sequences were subsequently processed as follows. Chimeric sequences were filtered out by Chimera-Slayer [42]. Average qual 25 was used as the minimal quality score to remove low quality reads. Qualifying sequences were further classified by the Ribosomal Database Project Naive Bayesian Classifier version 2.2 using training set 6 [43] from phylum to genus levels. Taxa assigned $<0.5$ confidence were reassigned to the next higher taxonomic level in which the classification threshold was $>0.5$.

Shotgun sequences from posterior fornix, tongue dorsum, supragingival plaque, anterior nares, stool and buccal mucosa were used to confirm the community classes identified by $16 \mathrm{~S}$ rRNA gene sequencing. Shotgun data were processed by the HMP consortium [39], resulting in measurement of depth and breadth of microbiota based on the reference database [44]. The WGS sequences can be downloaded from [45] and at NCBI at [46].

\section{Identification of community classes}

To cluster subjects with similar bacterial composition in sets of metagenomic samples, we followed the following statistical recipe. First, Ribosomal Database Project data are organized in a matrix format with rows being the subjects, columns being the genera, and entries in the table being the number of reads for that subject by genus combination. These read counts are scaled by dividing the number of reads belonging to that genus by the average copy number of $16 \mathrm{~S}$ rRNA genes for species belonging to that genus [47]. This was done to avoid overcounting genera with high gene copy numbers. The scaled counts were then transformed to percentages by dividing each count by the total number of scaled counts for that subject. Second, the proximity matrix used for the cluster analysis is built using the Bray-Curtis dissimilarity measure as a pairwise distance between the genera composition of subjects. The Bray-Curtis dissimilarity measure, $d(i, j)$, quantifies the dissimilarity in species composition between samples $i$ and $j$, based on the taxa abundances at each sample, and is defined as:

$$
d(i, j)=\frac{\sum_{k=1}^{n}\left|y_{i, k}-y_{j, k}\right|}{\sum_{k=1}^{n}\left(y_{i, k}+y_{j, k}\right)},
$$

where $y_{i, k}$ and $y_{j, k}$ are the abundances (in our case proportions) of genus $k$ in samples $i$ and $j$, respectively, and $n$ is total number of distinct genera present in both samples. The measure $d(i, j)$ ranges between 0 and 1 , where 0 means the two samples share all the genera in similar abundances, and 1 means the two subjects do not share any genera at all. This metric was chosen because it is commonly used in ecology because of its robust monotonic and linear relationship with ecological distance [48]. Third, the complete linkage criterion was used to form an agglomerative hierarchical clustering and dendrogram tree for identifying clusters [6]. The complete linkage criterion is a method to calculate distances between two clusters, which is defined as the distance between their most dissimilar members. While there are several criteria for agglomerative clustering, we choose this algorithm because it tends to produce compact clusters. Fourth, to determine the optimal number of clusters within a dendrogram, we used the Silhouette method [49]. The silhouette width $s(i)$ for each observation $\mathrm{j}$ is defined as:

$$
s(i):=(b(i)-a(i)) / \max (a(i), b(i))
$$

where $a(i)$ is the average dissimilarity between $i$ and all other points of the cluster to which $i$ belongs and $b(i)$, the dissimilarity between $i$ and its nearest cluster to which it does not belong. Observations with a large $s(i)$ (almost 1 ) are very well clustered, a small $s(i)$ (around 0 ) means that the observation lies between two clusters, and observations with a negative $s(i)$ are probably placed in the wrong cluster [50].

Averaged $s(i)$ for all the members of the clusters was used to assess the overall cluster quality. The number of clusters that yield the highest silhouette value was chosen to be the optimal number of clusters. Lastly, clusters with similar average silhouette values $(<0.02$ difference) were manually inspected. We have followed two general rules in this process: (a) within each cluster, silhouette values for the majority of the subjects were high this ensures the subjects with high similarity are grouped together and avoid heterogeneity within a cluster; (b) clusters with fewer than two samples were removed and cluster analysis was redone with the same procedure. This ensures the clusters we identified are sufficiently representative within a population.

We also performed fuzzy k-means clustering [50], using the same data matrix and dissimilarity measurement as described in hierarchical clustering.

\section{Principal coordinate analysis (PCoA)}

To illustrate the community classes identified in the human habitats, we performed PCoA analysis with the ade4 packages [51] in R. This starts with the Bray-Curtis 
dissimilarity matrix generated in the clustering analysis and assigns for each sample a location in a two-dimensional space. S class function was used to add additional variables into the graphs as indicated by the community classes with different colors. Cellipse indicating the inertia ellipse size was set to 1.5 .

\section{Indicator genera}

Indicator values were calculated using the DufreneLegendre Indicator Species approach from the labdsv [25] package in $\mathrm{R}$. The sample frequency (f) and relative average abundance (a) of each genus were calculated as follows:

p_\{i,j\} = presence/absence $(1 / 0)$ of species $\mathrm{i}$ in sample $\mathrm{j}$; $x_{-}\{i, j\}=$ abundance of species $i$ in sample $j$;

$\mathrm{n} \_\mathrm{c}=$ number of samples in cluster $\mathrm{c}$; ? for cluster $\mathrm{c}$ in set K;

$f \_\{i, c\}=\left\{\sum_{-}\{j \backslash\right.$ in $c\}$ p_ $\{i, j\}$ lover n_c $\}$

a_ $\{\mathrm{i}, \mathrm{c}\}=\left\{\left(\sum_{-}\{\mathrm{j} \backslash\right.\right.$ in $\left.\mathrm{c}\} \mathrm{x} \_\{\mathrm{i}, \mathrm{j}\}\right) / \mathrm{n} \_\mathrm{c}$ lover $\sum_{-}\{\mathrm{k}=1\}^{\wedge} \mathrm{K}$

$\left.\left(\left(\sum \_[j 48] x \_\{i, j\}\right) / n \_k\right)\right\}$

d_ $\{i, c\}=f \_\{i, c\} \backslash$ times a_ $\{i, c\}$

In this analysis, indicator genera were chosen based on: (1) indicator $P$-value $<0.01$; (2) genera present in at least $50 \%$ of subjects in either cluster.

\section{Quantifying the agreement of clusters between habitats and between visits}

The ARI was recommended for measurement of the agreement between two partitions in the clustering analysis after comparing many different indices [52]. The ARI is derived from the Rand Index and is the corrected-for-chance version of this index. It was computed by the fossil package in $\mathrm{R}$ [53]. Detailed information can be found in [54]. We used the ARI to compare the cluster similarity between different habitats. Clustering was performed as described above using the samples present in both compared habitats. To evaluate the stability of community class over time, clustering used all data from sampling times 1 and 2.

\section{Measurement of Shannon diversity}

The Shannon index was used to calculate alpha diversity. The samples were first rarified to 1,000 reads by the rarefy function in vegan [55] to prevent the bias caused by different read depth. Shannon diversity was calculated using the BiodiversityR package [56] as described below:

$$
H^{\prime}=-\sum_{i=1}^{S}\left(p_{i} \ln p_{i}\right)
$$

where $S$ is the number of species, and $p_{i}$ is the relative abundance of each species, calculated as the proportion of individuals of a given species to the total number of individuals in the community.

\section{Association of demographic factors with community classes and single taxa}

Demographic factors were mapped to the dendrogram from the hierarchical clustering [57]. The distribution of different of geographical locations (St Louis, Houston), gender (male, female), race and ethnicity (Hispanic/Latino/ Spanish, not Hispanic/Latino/Spanish) and BMI (BMI $<25$, $25 \leq \mathrm{BMI}<30$, BMI $\geq 30$ ) between community classes were assessed using Fisher's exact test. The ANOVA test was used when the data were continuous (age). Association of single taxa with gender was assessed by Mann-WhitneyWilcoxon test. $P$-values from multiple comparisons were corrected using the Bonferroni method. P-values $<0.05$ after correction were considered as significant.

\section{Data access}

The 16S rRNA gene sequences used are the July 2010 16S rRNA gene sequencing data freeze, 7,518 SRA runs, the Human Microbiome Project 16S rRNA Clinical Production Phase I. It is available from NCBI at [40], and from the HMP Data Analysis and Coordinating Center at [41]. The WGS sequences can be downloaded from [45] and at NCBI at [46]. Metadata were downloaded from dbGAP (study accession phs000228.v2.p1) [58].

\section{Additional files}

Additional file 1: Tables $\mathrm{S} 1$ to $\mathrm{S} 3$ and the figure legends for Figures S1 to S8.

Additional file 2: Figures S1 to S8.

\section{Abbreviations}

ARI: Adjusted Rand Index; BMI: body mass index; HMP: Human Microbiome Project; PCoA: principal coordinate analysis; WGS: whole genome shotgun.

\section{Competing interests}

The authors declare that they have no competing interests.

\section{Authors' contributions}

YZ, KAM, HG, PSLA, WDS, GMW, and ES: analysis and interpretation of data. YZ, KAM, HG, PSLA, WDS, MM, GMW, and ES: study design and concept. KMW: dbGap metadata processing. JCM, KK, and MM: WGS data processing $Y Z$ and GMW: drafting the paper. All authors read and approved the final manuscript.

\section{Acknowledgments}

The authors are grateful to the NIH Common Fund Human Microbiome Project Consortium for generating and making available the data sets used in this study, and Dr Phillip Tarr for critical reading and helpful comments on the manuscript. The research leading to these results received funding from the National Institutes of Health, grant U54HG004968 to GMW.

\section{Author details}

${ }^{1}$ The Genome Institute, Washington University, St Louis, MO 63108, USA. ${ }^{2}$ Medical School of Indiana University at Indianapolis, Indianapolis, IN 46202, USA. ${ }^{3}$ Washington University School of Medicine, Biostatistics in Medicine, St Louis, MO 63110, USA. ${ }^{4}$ Department of Pediatrics, Washington University 
School of Medicine, St Louis, MO 63110, USA. ${ }^{5}$ Current address: The Jackson Laboratory for Genomic Medicine, c/o University of Connecticut Health Center, 263 Farmington Avenue. Administrative Services Building, Call Box 901, Farmington, CT 06030, USA.

Received: 9 September 2013 Accepted: 7 May 2014 Published: 7 May 2014

\section{References}

1. Dewhirst FE, Chen T, Izard J, Paster BJ, Tanner AC, Yu WH, Lakshmanan A, Wade WG: The human oral microbiome. J Bacteriol 2010, 192:5002-5017.

2. Eckburg PB, Bik EM, Bernstein CN, Purdom E, Dethlefsen L, Sargent M, Gill $\mathrm{SR}$, Nelson KE, Relman DA: Diversity of the human intestinal microbial flora. Science 2005, 308:1635-1638.

3. Fierer $\mathrm{N}$, Hamady M, Lauber CL, Knight R: The influence of sex, handedness, and washing on the diversity of hand surface bacteria. Proc Natl Acad Sci US A 2008, 105:17994-17999.

4. Grice EA, Kong HH, Conlan S, Deming CB, Davis J, Young AC, Bouffard GG, Blakesley RW, Murray PR, Green ED, Turner ML, Segre JA: Topographical and temporal diversity of the human skin microbiome. Science 2009, 324:1190-1192.

5. Ley RE, Turnbaugh PJ, Klein S, Gordon J: Microbial ecology: human gut microbes associated with obesity. Nature 2006, 444:1022-1023

6. Ravel J, Gajer P, Abdo Z, Schneider GM, Koenig SS, McCulle SL, Karlebach S, Gorle R, Russell J, Tacket CO, Brotman RM, Davis CC, Ault K, Peralta L, Forney $\sqcup$ : Vaginal microbiome of reproductive-age women. Proc Natl Acad Sci U S A 2011, 108:4680-4687.

7. Costello EK, Lauber CL, Hamady M, Fierer N, Gordon Jl, Knight R: Bacterial community variation in human body habitats across space and time. Science 2009, 326:1694-1697.

8. The Human Microbiome Consortium: Structure, function and diversity of the human microbiome in an adult reference population. Nature 2012, 486:207-214.

9. Huse $S M, Y e Y$, Zhou $Y$, Fodor AA: A core human microbiome as viewed through 16S rRNA sequence clusters. PLoS One 2012, 7:e34242.

10. Gajer P, Brotman RM, Bai G, Sakamoto J, Schutte UM, Zhong X, Koenig SS, Fu L, Ma ZS, Zhou X, Zhou X, Abdo Z, Forney LJ, Ravel J: Temporal dynamics of the human vaginal microbiota. Sci Trans/ Med 2012, 4:132ra152.

11. Arumugam M, Raes J, Pelletier E, Le Paslier D, Yamada T, Mende DR, Fernandes GR, Tap J, Bruls T, Batto JM, Bertalan M, Borruel N, Casellas F, Fernandez L, Gautier L, Hansen T, Hattori M, Hayashi T, Kleerebezem M, Kurokawa K, Leclerc M, Levenez F, Manichanh C, Nielsen HB, Nielsen T, Pons N, Poulain J, Qin J, Sicheritz-Ponten T, Tims S: Enterotypes of the human gut microbiome. Nature 2011, 473:174-180.

12. Wu GD, Chen J, Hoffmann C, Bittinger $K$, Chen YY, Keilbaugh SA, Bewtra M Knights D, Walters WA, Knight R, Sinha R, Gilroy E, Gupta K, Baldassano R, Nessel L, Li H, Bushman FD, Lewis JD: Linking long-term dietary patterns with gut microbial enterotypes. Science 2011, 334:105-108.

13. Zupancic ML, Cantarel BL, Liu Z, Drabek EF, Ryan KA, Cirimotich S, Jones C, Knight R, Walters WA, Knights D, Mongodin EF, Horenstein RB, Mitchell BD, Steinle N, Snitker S, Shuldiner AR, Fraser CM: Analysis of the gut microbiota in the old order Amish and its relation to the metabolic syndrome. PLOS One 2012, 7:e43052

14. Moeller AH, Degnan PH, Pusey AE, Wilson ML, Hahn BH, Ochman H: Chimpanzees and humans harbour compositionally similar gut enterotypes. Nat Commun 2012, 3:1179.

15. Hildebrand F, Nguyen AT, Brinkman B, Yunta RG, Cauwe B, Vandenabeele $P$, Liston A, Raes J: Inflammation-associated enterotypes, host genotype, cage and inter-individual effects drive gut microbiota variation in common laboratory mice. Genome Biol 2013, 14:R4.

16. Jeffery IB, Claesson MJ, O'Toole PW, Shanahan F: Categorization of the gut microbiota: enterotypes or gradients? Nat Rev Microbiol 2012, 10:591-592.

17. Koren O, Knights D, Gonzalez A, Waldron L, Segata N, Knight R, Huttenhower C, Ley RE: A guide to enterotypes across the human body: meta-analysis of microbial community structures in human microbiome datasets. PLoS Comput Biol 2013, 9:e1002863.

18. Yatsunenko T, Rey FE, Manary MJ, Trehan I, Dominguez-Bello MG, Contreras M, Magris M, Hidalgo G, Baldassano RN, Anokhin AP, Heath AC, Warner B, Reeder J, Kuczynski J, Caporaso JG, Lozupone CA, Lauber C, Clemente JC,
Knights D, Knight R, Gordon J: Human gut microbiome viewed across age and geography. Nature 2012, 486:222-227.

19. Goldstein DR, Ghosh D, Conlon EM: Statistical issues in the clustering of gene expression data. Stat $\operatorname{Sin} 2002,12: 219-240$.

20. Richards AL, Holmans P, O'Donovan MC, Owen MJ, Jones L: A comparison of four clustering methods for brain expression microarray data. BMC Bioinforma 2008, 9:490

21. Thalamuthu A, Mukhopadhyay I, Zheng X, Tseng GC: Evaluation and comparison of gene clustering methods in microarray analysis. Bioinformatics 2006, 22:2405-2412.

22. Calinski T, Harabasz J: A dendrite method for cluster analysis. Commun Stat 1974, 3:1-27.

23. Zhou Y, Gao H, Mihindukulasuriya KA, Rosa PS, Wylie KM, Vishnivetskaya T, Podar M, Warner B, Tarr PI, Nelson DE, Fortenberry JD, Holland MJ, Burr SE, Shannon WD, Sodergren E, Weinstock GM: Biogeography of the ecosystems of the healthy human body. Genome Biol 2013, 14:R1.

24. Dufrêne $M$, Legendre $P$ : Species assemblages and indicator species: the need for a flexible asymmetrical approach. Ecol Monogr 1997, 67:345-366.

25. labdsv: Ordination and Multivariate Analysis for Ecology. [http://CRAN R-project.org/package=labdsv]

26. Fredricks DN, Fiedler TL, Marrazzo JM: Molecular identification of bacteria associated with bacterial vaginosis. N Engl J Med 2005, 353:1899-1911.

27. Wos-Oxley ML, Plumeier I, von Eiff C, Taudien S, Platzer M, Vilchez-Vargas R, Becker K, Pieper DH: A poke into the diversity and associations within human anterior nare microbial communities. ISME J 2010, 4:839-851.

28. Goto T, Yamashita A, Hirakawa H, Matsutani M, Todo K, Ohshima K, Toh H, Miyamoto K, Kuhara S, Hattori M, Shimizu T, Akimoto S: Complete genome sequence of Finegoldia magna, an anaerobic opportunistic pathogen. DNA Res 2008, 15:39-47.

29. Tanner A, Lai CH, Maiden M: Characteristics of oral gram-negative species. In Contemporary Oral Microbiology and Immunology. Edited by Slots J, Taubman MA. St Louis: Mosby-Year Book: 1992:299-341.

30. Kenney EB, Ash MM Jr: Oxidation reduction potential of developing plaque, periodontal pockets and gingival sulci. J Periodontol 1969, 40:630-633.

31. Wagner S, Wagner D: Comparing clusterings - an overview. In Technical Report 2006-04. Karlsruhe: Universit at Karlsruhe; 2007

32. Aagaard K, Petrosino J, Keitel W, Watson M, Katancik J, Garcia N, Patel S, Cutting M, Madden T, Hamilton H, Harris E, Gevers D, Simone G, McInnes P, Versalovic J: The Human Microbiome Project strategy for comprehensive sampling of the human microbiome and why it matters. FASEB J 2013, 27:1012-1022

33. Rosenthal M, Goldberg D, Aiello A, Larson E, Foxman B: Skin microbiota: microbial community structure and its potential association with health and disease. Infect Genet Evol 2011, 11:839-848.

34. Troccaz M, Borchard G, Vuilleumier C, Raviot-Derrien S, Niclass Y, Beccucci S, Starkenmann C: Gender-specific differences between the concentrations of nonvolatile (R)/(S)-3-methyl-3-sulfanylhexan-1-Ol and (R)/(S)-3-hydroxy-3-methyl-hexanoic acid odor precursors in axillary secretions. Chem Senses 2009, 34:203-210.

35. Claesson MJ, Cusack S, O'Sullivan O, Greene-Diniz R, de Weerd H, Flannery E, Marchesi JR, Falush D, Dinan T, Fitzgerald G, Shanahan F, Twomey C, Hill C, Ross RP, O'Toole PW: Composition, variability, and temporal stability of the intestinal microbiota of the elderly. Proc Natl Acad Sci U S A 2011, 108:4586-4591.

36. Keane FE, Ison CA, Taylor-Robinson D: A longitudinal study of the vaginal flora over a menstrual cycle. Int J STD AIDS 1997, 8:489-494.

37. Eschenbach DA, Thwin SS, Patton DL, Hooton TM, Stapleton AE, Agnew K, Winter C, Meier A, Stamm WE: Influence of the normal menstrual cycle on vaginal tissue, discharge, and microflora. Clin Infect Dis 2000, 30:901-907.

38. Caporaso JG, Lauber CL, Costello EK, Berg-Lyons D, Gonzalez A, Stombaugh J, Knights D, Gajer P, Ravel J, Fierer N, Gordon Jl, Knight R: Moving pictures of the human microbiome. Genome Biol 2011, 12:R50.

39. The Human Microbiome Consortium: A framework for human microbiome research. Nature 2012, 486:215-221.

40. NCBI SRA: HMP 16s rRNA gene. [http://www.ncbi.n/m.nih.gov/sra/? term=SRP002395]

41. HMP DACC: HMR16S - Raw 16S Data and Library Metadata. [http://www hmpdacc.org/HMR16S/]

42. Haas BJ, Gevers D, Earl AM, Feldgarden M, Ward DV, Giannoukos G, Ciulla D, Tabbaa D, Highlander SK, Sodergren E, Methe B, DeSantis TZ, Petrosino JF, 
Knight R, Birren BW: Chimeric 16S rRNA sequence formation and detection in Sanger and 454-pyrosequenced PCR amplicons. Genome Res 2011, 21:494-504.

43. Cole JR, Wang Q, Cardenas E, Fish J, Chai B, Farris RJ, Kulam-Syed-Mohideen AS, McGarrell DM, Marsh T, Garrity GM, Tiedje JM: The Ribosomal Database Project: improved alignments and new tools for rRNA analysis. Nucleic Acids Res 2009, 37:D141-D145.

44. Martin J, Sykes S, Young S, Kota K, Sanka R, Sheth N, Orvis J, Sodergren E, Wang Z, Weinstock GM, Mitreva M: Optimizing read mapping to reference genomes to determine composition and species prevalence in microbial communities. PLoS One 2012, 7:e36427.

45. HMP DACC: HMSCP - Shotgun community profiling. [http://www. hmpdacc.org/HMSCP/]

46. NCBI BioProject: HMP shotgun data. [www.ncbi.nlm.nih.gov/bioproject/48479]

47. Lee ZM, Bussema $C$ 3rd, Schmidt TM: rrnDB: documenting the number of rRNA and tRNA genes in bacteria and archaea. Nucleic Acids Res 2009, 37:D489-D493.

48. Faith DP, Minchin PR, Belbin L: Compositional dissimilarity as a robust measure of ecological distance. Vegetatio 1987, 69:57-68.

49. Rousseeuw P: Silhouettes: a graphical aid to the interpretation and validation of cluster analysis. J Comput Appl Math 1987, 20:53-65.

50. Maechler M, Rousseeuw P, Struyf A, Hubert M, Hornik K: cluster: Cluster Analysis Basics and Extensions. 2013. R package version 1.14.4.

51. Dray S, Dufour AB: The ade4 package: implementing the duality diagram for ecologists. J Stat Softw 2007, 22:1-20.

52. Milligan GW, Cooper MC: A study of the comparability of external criteria for hierarchical cluster analysis. Multivar Behav Res 1986, 21:441-458.

53. Vavrek MJ: fossil: palaeoecological and palaeogeographical analysis tools. Palaeontol Electron 2011, 14:1T.

54. Hubert L, Arabie P: Comparing partitions. J Classif 1985, 2:193-218.

55. Jari O, Guillaume B, Roeland K, Pierre L, Peter RM, O'Hara RB, Gavin LS, Peter S, Henry HS, Helene W: vegan: Community Ecology Package. 2013, [http://CRAN.R-project.org/package=vegan]

56. Kindt R, Coe R: Tree Diversity Analysis. A Manual and Software for Common Statistical Methods for Ecological and Biodiversity Studies. World Agroforestry Centre (ICRAF). Nairobi; 2005.

57. Letunic I, Bork P: Interactive Tree Of Life (iTOL): an online tool for phylogenetic tree display and annotation. Bioinformatics 2007, 23:127-128.

58. HMP Metadata. [http://www.ncbi.nlm.nih.gov/gap/?term=phs000228.v3.p1]

doi:10.1186/gb-2014-15-5-r66

Cite this article as: Zhou et al.: Exploration of bacterial community classes in major human habitats. Genome Biology 2014 15:R66.

\section{Submit your next manuscript to BioMed Central and take full advantage of:}

- Convenient online submission

- Thorough peer review

- No space constraints or color figure charges

- Immediate publication on acceptance

- Inclusion in PubMed, CAS, Scopus and Google Scholar

- Research which is freely available for redistribution 